\title{
INTERAÇÃO SOLO-PLANTA AVALIADA POR MODELAGEM ESTATÍSTICA DE ESPAÇO DE ESTADOS
}

\author{
Luis Carlos Timm ${ }^{1,5,6 *}$; Lorival Fante Jr. ${ }^{2,5}$; Emanuel Pimentel Barbosa ${ }^{3}$; Klaus Reichardt+, ${ }^{4,5}$ Osny \\ Oliveira Santos Bacchi ${ }^{5}$ \\ ${ }^{1}$ Pós-Graduando em Irrigação e Drenagem, USP/ESALQ. \\ ${ }^{2}$ Fac. de Ciências Matemáticas e da Natureza - UNIMEP, C.P. 68 - CEP: 13400-911 - Piracicaba, SP. \\ ${ }^{3}$ Depto. de Estatística, IMECC/UNICAMP, C.P. 6065 - CEP: 13083-970 - Campinas, SP. \\ ${ }^{4}$ Depto. de Ciências Exatas, USP/ESALQ, C.P. 9 - CEP: 134148-900 - Piracicaba, SP. \\ ${ }^{5}$ Lab. Física do Solo - USP/CENA, C.P. 96 - CEP: 13400-970 - Piracicaba, SP. \\ ${ }^{6}$ Bolsista FAPESP. \\ *Autor correspondente <lctimm@carpa.ciagri.usp.br>
}

RESUMO: Para estudar a interação solo-planta utilizou-se um modelo de espaço de estados (regressão dinâmica) comparativamente à um modelo de regressão estática, em versão convencional e em versão sequencial. Para tal, foram utilizados dados experimentais de densidade do solo, macroporosidade, microporosidade e porosidade total de um solo Latossolo Vermelho Escuro, além da densidade radicular por comprimento de raiz por unidade de volume, de uma cultura de aveia forrageira. Das variáveis explanatórias estudadas, a porosidade total apresentou correlação bastante significativa com a densidade radicular, considerada como a variável resposta do estudo. Um modelo de regressão estática representado na forma de espaço de estados com estimação seqüencial forneceu um coeficiente $R^{2}$ de ajuste igual a 0.69 , enquanto que um modelo estático convencional forneceu um coeficiente $R^{2}$ de ajuste de apenas 0.59 . Esta relação soloplanta é melhor representada ainda através de um modelo de regressão dinâmica, ou seja, um modelo genuinamente de espaço de estados onde agora o grau de explicação $R^{2}$ passa a mais de 0.98 . Isto explicita a vantagem da abordagem de espaço de estados em relação aos outros métodos mais tradicionais de relacionar sistema solo-planta.

Palavras-chave: variabilidade espacial, regressão dinâmica, regressão estática, estimação seqüencial

\section{SOIL-PLANT INTERACTION EVALUATED BY THE STATE-SPACE APPROACH}

\begin{abstract}
The interaction soil-plant was evaluated using a state space approach (dynamic model) comparatively to a static regression model using both, standard and sequential estimations. Experimental soil data consisted of bulk density, macroporosity, microporosity and porosity of a dark red latosol, and plant data of root density in length per unit volume, of a forage-oat crop. Among these, only soil porosity had a good correlation with the root system density, which is the response variable of this study. A static regression model written in the state space form with a sequential estimation, gave a $R^{2}$ coefficient of 0.69 , comparatively to a conventional (non-sequential) regression model, which gave a $R^{2}$ coefficient of only 0.59 . This soil-plant relation was better described by a dynamic regression model, which gave a $R^{2}$ coefficient greater than 0.98 . These results indicate the advantage of the state space approach in relation to the other more conventional regression methods.

Key words: spatial variability, dynamic regression, static regression, sequential estimation
\end{abstract}

\section{INTRODUÇÃO}

A caracterização da distribuição do sistema radicular de uma cultura agrícola pode ser entendida como uma prática fundamental em estudos sobre a eficiência de sistemas de manejo. Estes visam a otimização da produtividade agrícola num contexto de sustentabilidade e conservação dos agentes integrantes no sistema solo-planta-atmosfera. Esta distribuição resulta de uma série de processos complexos e dinâmicos que se interrelacionam com os atributos físicos, químicos e biológicos do solo. Segundo Brown \& Scott (1984), as condições que determinam o crescimento das raízes e sua forma de distribuição, encontram-se nas características e propriedades do solo na zona radicular, como a densidade e porosidade do solo, o movimento de água, os nutrientes, o pH e a presença de elementos tóxicos, dentre outros.

Vários trabalhos têm destacado a densidade e a porosidade do solo como os principais agentes responsáveis na interação solo-raiz, a partir de relações existentes entre estes atributos físicos do solo e o crescimento de raízes (Hatano et al., 1988; Fante Jr, 1997; Salih et al., 1998; Merotto Jr. \& Mundstock, 1999). Nestes trabalhos, foram verificadas correlações negativas entre a densidade do solo e a presença de raízes, assim como, correlações entre esta presença radicular e a porosidade do solo. 
Existem vários métodos que permitem investigar a distribuição do sistema radicular (Bohm, 1979; Kopke, 1981; Crestana et al., 1994), mas a avaliação das raízes em termos de volume explorado, massa seca e comprimento radicular é tarefa difícil e grandes dificuldades são encontradas em qualquer técnica, como tempo gasto, a pouca informação obtida e a variabilidade dos resultados. Da mesma forma, a variabilidade espacial e temporal do sistema radicular de uma cultura agrícola também pode ser considerada como um fator limitante em estudos sobre as relações entre o crescimento radicular e os atributos do solo (Shibo Hui et al., 1998; Fante Jr. et al., 1999). Em função destas dificuldades na análise e na interpretação da arquitetura das raízes e de sua relação com os componentes do solo, diversos estudos apontam para o desenvolvimento de novas técnicas, novas idéias e abordagens, que permitam a obtenção mais detalhada e abrangente da interface solo-raiz (Longsdon \& Linden, 1992; Teruel, 1999).

Os problemas relativos aos efeitos da variabilidade espacial e temporal da distribuição das raízes, tem levado muitos pesquisadores a enfatizarem o emprego de uma abordagem geoestatística centrada em uma dependência espacial e/ou temporal, utilizando-se da aplicação de modelos e técnicas de séries temporais e/ou espaciais, como por exemplo uma análise desses dados através de modelos de espaço de estados (Morkoc et al., 1985; Shumway, 1988; Parlange et al., 1992; Wendroth et al., 1992; Dourado et al., 1999).

Neste contexto, o presente trabalho teve como proposta a aplicação da análise de espaço de estados através de um modelo de regressão dinâmica (West \& Harrison, 1989, 1997; Barbosa, 1991) para verificar possíveis relações significativas entre a distribuição do sistema radicular de uma cultura agrícola e os atributos físicos do solo. Além disso, é mostrada a vantagem de tais modelos dinâmicos de espaço de estados em relação a sua contrapartida estática mais convencional, no que diz respeito a uma melhor explicação da interrelação dos atributos solo-planta.

Neste estudo foram utilizados dados de comprimento de raiz e dos seguintes atributos físicos do solo: densidade do solo, macroporosidade, microporosidade e porosidade total do solo. Estes dados foram extraídos de Fante Jr. (1997).

A relação entre o comprimento da raiz e os atributos físicos do solo é representada através de modelos de regressão, porém não de uma forma estática com coeficientes fixos, mas sim de uma forma dinâmica com coeficientes variáveis no espaço através de representação de espaço de estados.

Nas sessões seguintes é dada ênfase à descrição de uma forma sucinta da metodologia utilizada na coleta dos dados experimentais; apresentação destes dados em forma de TABELA; análise descritiva e análise via modelos de espaço de estados (com sua comparação com modelos de regressão tradicional); os resultados obtidos e a discussão destes; e, por último, as referências bibliográficas relacionadas.

\section{MATERIAL E MÉTODOS}

\section{Dados experimentais}

Os dados analisados (TABELAS 1, 2 e 3) são experimentais e foram obtidos por Fante Jr (1997) em campo experimental da Fazenda Canchim da EMBRAPA/CPPSE de São Carlos (SP), em área de Latossolo Vermelho Escuro de classe textural franco argiloso, cultivada com aveia forrageira, variedade São Carlos. Na avaliação do sistema radicular da aveia forrageira e na determinação dos atributos densidade e porosidade do solo, foi empregado o método do anel volumétrico (Bohm, 1979; Kiehl, 1979; Embrapa, 1997). A partir da abertura de uma trincheira, foram retirados do perfil do solo, 25 anéis separados entre si por aproximadamente $0.1 \mathrm{~m}$, em camadas de $0.1 \mathrm{~m}$ da superfície até $0.3 \mathrm{~m}$ de profundidade, totalizando 75 amostras indeformadas. Após retirada do perfil, cada amostra de solo foi moldada segundo o volume do anel (diâmetro de $0.046 \mathrm{~m}$ e altura de $0.030 \mathrm{~m}$ ), saturada em água, pesada e logo em seguida colocada em mesa de tensão sob a pressão de $-0.60 \mathrm{~m}$ de água durante 48 horas. Retirada da mesa de tensão, cada amostra foi pesada, colocada em estufa à $105{ }^{\circ} \mathrm{C}$ por 48 horas e novamente pesada. Com este procedimento determinaram-se os atributos densidade, macro, micro e porosidade do solo.

A separação das raízes das amostras indeformadas de solo, se deu por dispersão em água com agitação mecânica e utilização de peneira de malha 0,2 $\mathrm{mm}$. Depois de separadas, as raízes foram colocadas em um recipiente contendo água sobre um "scanner" de mesa, para a obtenção de imagens digitalizadas. O processamento dessas imagens, para a determinação do comprimento radicular, foi feito utilizando o programa computacional SIARCS (Crestana et al., 1994; Fante Jr., 1997). Na quantificação das raízes utilizou-se da grandeza densidade radicular por comprimento de raiz $(\mathrm{DRC}=$ comprimento radicular / volume da amostra indeformada de solo).

\section{Análise descritiva dos dados}

A partir dos dados apresentados nas TABELAS 1,2 e 3 foram calculados os coeficientes de correlação linear entre a variável de interesse de estudo, dita resposta (DRC) e as variáveis tomadas como explicativas, ou seja, densidade do solo, macroporosidade e porosidade total do solo, além da porosidade total defasada de 1 espaçamento (porosidade no ponto vizinho). Os coeficientes de correlação encontrados foram $-0.62,0.70,0.63$ e 0.61 , respectivamente. Também constatou-se que a microporosidade não apresentou um coeficiente de correlação expressivo, logo, esta variável não foi incluída no estudo. Nas Figuras 1, 2, 3 e 4 abaixo são apresentados os diagramas de dispersão das variáveis densidade do solo, macroporosidade, porosidade total do solo e porosidade total defasada de 1 espaçamento, todas em função da variável DRC. A variável porosidade defasada de um 1 espaçamento, também apresentou uma correlação satisfatória, o que não aconteceu com as outras duas variáveis quando defasadas. 
TABELA 1 - Densidade radicular por comprimento de raiz (DRC), densidade do solo (Ds), microporosidade (Mip), macroporosidade (Map) e porosidade total do solo $(P)$ na camada de $0-0.10 \mathrm{~m}$ de profundidade, obtidos por Fante Jr. (1997).

\begin{tabular}{|c|c|c|c|c|c|c|}
\hline & & DRC & Ds & Mip & Map & $P$ \\
\hline \multirow[t]{13}{*}{ Camada } & Pontos & $10^{4} \mathrm{~m} \mathrm{~m}^{-3}$ & $\left(10^{3} \mathrm{~kg} \mathrm{~m}^{-3}\right.$ & ----------- & $\mathrm{m}^{3} \mathrm{~m}^{-3}$ & \\
\hline & 1 & 8.92 & 1.22 & 0.39 & 0.18 & 0.57 \\
\hline & 2 & 1.53 & 1.28 & 0.40 & 0.15 & 0.55 \\
\hline & 3 & 7.40 & 1.28 & 0.38 & 0.19 & 0.57 \\
\hline & 4 & 3.82 & 1.22 & 0.39 & 0.20 & 0.59 \\
\hline & 5 & 4.32 & 1.13 & 0.37 & 0.23 & 0.60 \\
\hline & 6 & 4.68 & 1.29 & 0.39 & 0.16 & 0.55 \\
\hline & 7 & 1.68 & 1.27 & 0.37 & 0.16 & 0.53 \\
\hline & 8 & 4.18 & 1.23 & 0.38 & 0.22 & 0.60 \\
\hline & 9 & 2.48 & 1.19 & 0.38 & 0.18 & 0.56 \\
\hline & 10 & 2.67 & 1.21 & 0.37 & 0.23 & 0.60 \\
\hline & 11 & 5.69 & 1.28 & 0.42 & 0.19 & 0.61 \\
\hline & 12 & 4.71 & 1.33 & 0.40 & 0.19 & 0.59 \\
\hline \multirow[t]{13}{*}{$0-0.10 \mathrm{~m}$} & 13 & 10.10 & 1.23 & 0.38 & 0.22 & 0.60 \\
\hline & 14 & 4.80 & 1.18 & 0.39 & 0.22 & 0.61 \\
\hline & 15 & 8.80 & 1.16 & 0.36 & 0.29 & 0.65 \\
\hline & 16 & 7.05 & 1.19 & 0.38 & 0.22 & 0.60 \\
\hline & 17 & 6.38 & 1.27 & 0.39 & 0.20 & 0.59 \\
\hline & 18 & 5.63 & 1.31 & 0.40 & 0.18 & 0.58 \\
\hline & 19 & 5.41 & 1.06 & 0.34 & 0.26 & 0.60 \\
\hline & 20 & 7.06 & 1.29 & 0.40 & 0.20 & 0.60 \\
\hline & 21 & 5.64 & 1.30 & 0.42 & 0.21 & 0.63 \\
\hline & 22 & 5.66 & 1.41 & 0.23 & 0.20 & 0.43 \\
\hline & 23 & 4.04 & 1.26 & 0.42 & 0.19 & 0.61 \\
\hline & 24 & 9.25 & 1.22 & 0.39 & 0.19 & 0.58 \\
\hline & 25 & 4.00 & 1.23 & 0.39 & 0.20 & 0.59 \\
\hline
\end{tabular}

TABELA 2 - Densidade radicular por comprimento de raiz (DRC), densidade do solo (Ds), microporosidade (Mip), macroporosidade (Map) e porosidade total do solo (P) na camada de $0.10-0.20 \mathrm{~m}$ de profundidade, obtidos por Fante Jr. (1997).

\begin{tabular}{|c|c|c|c|c|c|c|}
\hline & & DRC & Ds & Mip & Map & $P$ \\
\hline \multirow{13}{*}{ Camada } & Pontos & $10^{4} \mathrm{~m} \mathrm{~m}^{-3}$ & $10^{3} \mathrm{~kg} \mathrm{~m}^{-3}$ & - & $n^{3} m^{-3}$ & $\cdots$ \\
\hline & 26 & 5.52 & 1.44 & 0.44 & 0.08 & 0.52 \\
\hline & 27 & 2.57 & 1.52 & 0.42 & 0.09 & 0.51 \\
\hline & 28 & 1.50 & 1.45 & 0.42 & 0.13 & 0.55 \\
\hline & 29 & 1.96 & 1.37 & 0.40 & 0.13 & 0.53 \\
\hline & 30 & 2.04 & 1.42 & 0.42 & 0.11 & 0.53 \\
\hline & 31 & 0.92 & 1.51 & 0.46 & 0.05 & 0.51 \\
\hline & 32 & 1.47 & 1.44 & 0.44 & 0.08 & 0.52 \\
\hline & 33 & 1.73 & 1.41 & 0.42 & 0.13 & 0.55 \\
\hline & 34 & 1.14 & 1.49 & 0.44 & 0.08 & 0.52 \\
\hline & 35 & 0.88 & 1.51 & 0.44 & 0.09 & 0.53 \\
\hline & 36 & 1.71 & 1.42 & 0.42 & 0.10 & 0.52 \\
\hline & 37 & 1.07 & 1.51 & 0.42 & 0.09 & 0.51 \\
\hline \multirow[t]{13}{*}{$0.10-0.20 \mathrm{~m}$} & 38 & 2.48 & 1.40 & 0.45 & 0.11 & 0.56 \\
\hline & 39 & 1.78 & 1.43 & 0.42 & 0.11 & 0.53 \\
\hline & 40 & 0.99 & 1.38 & 0.42 & 0.11 & 0.53 \\
\hline & 41 & 3.78 & 1.49 & 0.46 & 0.04 & 0.50 \\
\hline & 42 & 3.52 & 1.42 & 0.46 & 0.10 & 0.56 \\
\hline & 43 & 1.92 & 1.40 & 0.42 & 0.11 & 0.53 \\
\hline & 44 & 2.51 & 1.45 & 0.46 & 0.09 & 0.55 \\
\hline & 45 & 1.93 & 1.34 & 0.44 & 0.12 & 0.56 \\
\hline & 46 & 1.92 & 1.40 & 0.44 & 0.08 & 0.52 \\
\hline & 47 & 0.91 & 1.44 & 0.40 & 0.09 & 0.49 \\
\hline & 48 & 2.45 & 1.33 & 0.38 & 0.16 & 0.54 \\
\hline & 49 & 1.59 & 1.38 & 0.42 & 0.10 & 0.52 \\
\hline & 50 & 2.30 & 1.42 & 0.42 & 0.10 & 0.52 \\
\hline
\end{tabular}


TABELA 3 - Densidade radicular por comprimento de raiz (DRC), densidade do solo (Ds), microporosidade (Mip), macroporosidade (Map) e porosidade total do solo (P) na camada de $0.20-0.30 \mathrm{~m}$ de profundidade, obtidos por Fante Jr. (1997).

\begin{tabular}{|c|c|c|c|c|c|c|}
\hline & & DRC & Ds & Mip & Map & $P$ \\
\hline \multirow[t]{13}{*}{ Camada } & Pontos & $10^{4} \mathrm{~m} \mathrm{~m}^{-3}$ & $10^{3} \mathrm{~kg} \mathrm{~m}^{-3}$ & (-n- & $m^{3} m^{-3}$ & - \\
\hline & 51 & 0.86 & 1.50 & 0.41 & 0.12 & 0.53 \\
\hline & 52 & 1.30 & 1.40 & 0.41 & 0.09 & 0.50 \\
\hline & 53 & 1.75 & 1.38 & 0.42 & 0.14 & 0.56 \\
\hline & 54 & 0.38 & 1.31 & 0.37 & 0.12 & 0.49 \\
\hline & 55 & 0.78 & 1.38 & 0.41 & 0.12 & 0.53 \\
\hline & 56 & 1.77 & 1.45 & 0.42 & 0.11 & 0.53 \\
\hline & 57 & 1.04 & 1.40 & 0.40 & 0.12 & 0.52 \\
\hline & 58 & 0.95 & 1.40 & 0.41 & 0.11 & 0.52 \\
\hline & 59 & 1.11 & 1.41 & 0.42 & 0.11 & 0.53 \\
\hline & 60 & 1.71 & 1.37 & 0.42 & 0.17 & 0.59 \\
\hline & 61 & 1.03 & 1.36 & 0.39 & 0.16 & 0.55 \\
\hline & 62 & 0.81 & 1.47 & 0.42 & 0.09 & 0.51 \\
\hline \multirow[t]{13}{*}{$0.20-0.30 \mathrm{~m}$} & 63 & 0.28 & 1.38 & 0.42 & 0.11 & 0.53 \\
\hline & 64 & 0.83 & 1.44 & 0.42 & 0.10 & 0.52 \\
\hline & 65 & 0.59 & 1.44 & 0.40 & 0.08 & 0.48 \\
\hline & 66 & 0.41 & 1.40 & 0.38 & 0.10 & 0.48 \\
\hline & 67 & 0.83 & 1.45 & 0.42 & 0.11 & 0.53 \\
\hline & 68 & 1.21 & 1.50 & 0.42 & 0.12 & 0.54 \\
\hline & 69 & 1.30 & 1.35 & 0.40 & 0.13 & 0.53 \\
\hline & 70 & 1.00 & 1.38 & 0.41 & 0.14 & 0.55 \\
\hline & 71 & 0.83 & 1.42 & 0.42 & 0.10 & 0.52 \\
\hline & 72 & 0.32 & 1.32 & 0.38 & 0.13 & 0.51 \\
\hline & 73 & 4.10 & 1.38 & 0.40 & 0.14 & 0.54 \\
\hline & 74 & 1.22 & 1.36 & 0.41 & 0.12 & 0.53 \\
\hline & 75 & 1.10 & 1.33 & 0.38 & 0.14 & 0.52 \\
\hline
\end{tabular}

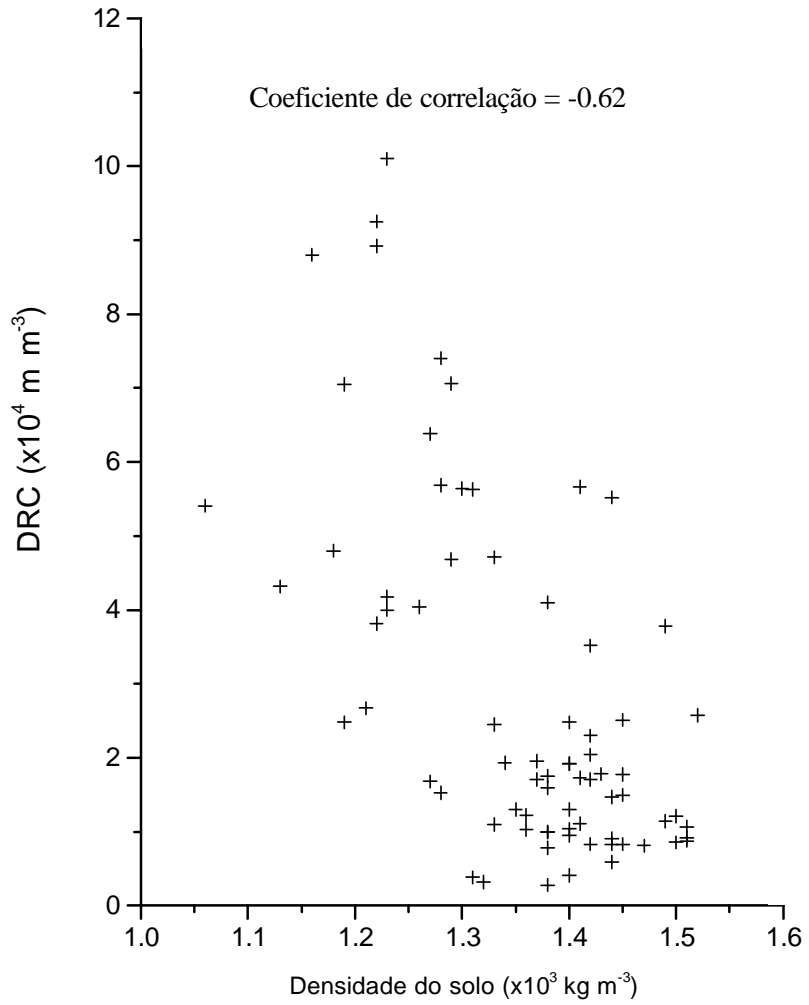

Figura 1- A dispersão dos dados de comprimento de raiz em função da densidade do solo, apresentando um coeficiente de correlação (r) igual a -0.62 .

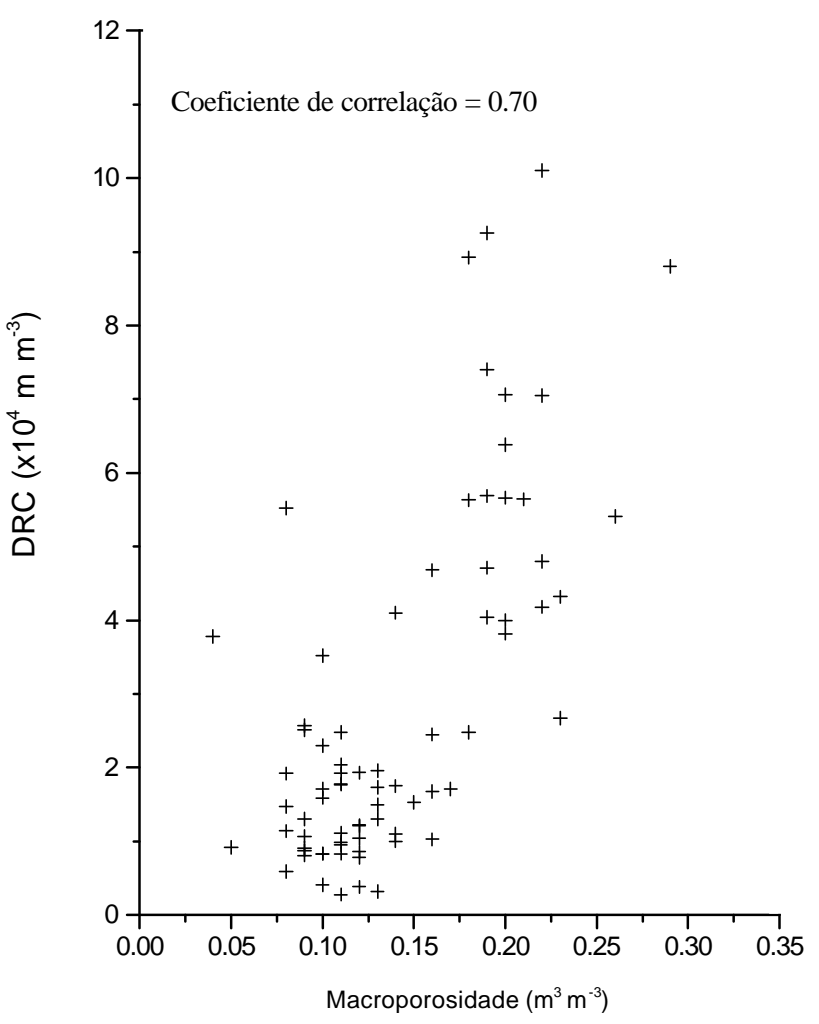

Figura 2 - A dispersão dos dados de comprimento de raiz em função da macroporosidade do solo, apresentando um coeficiente de correlação (r) igual a 0.70 . 


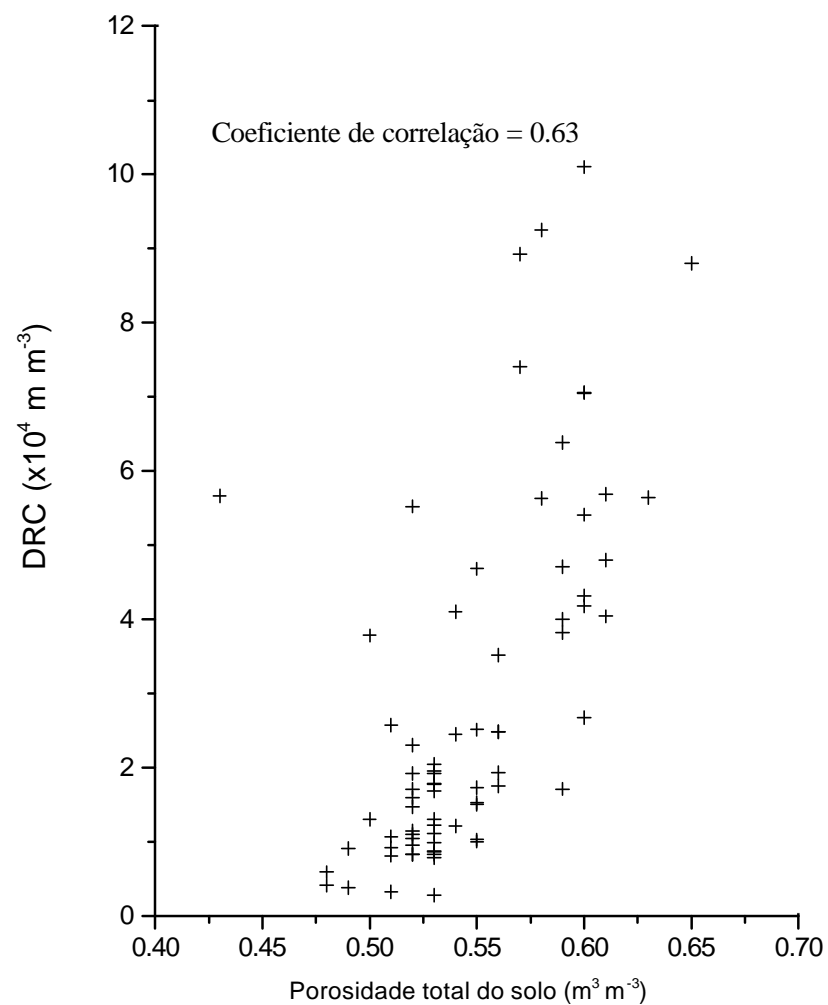

Figura 3 - A dispersão dos dados de comprimento de raiz em função da porosidade total do solo, apresentando um coeficiente de correlação (r) igual a 0.63 .

Em seguida, foi feita uma análise da normalidade dos dados de DRC (utilizando-se de histograma e gráfico 'normal plot'), com o intuito de verificar se havia necessidade de algum tipo de transformação, já que a etapa seguinte que é a de análise via modelos, pressupõem a normalidade dos dados. Foi constatado que a variável DRC não apresentava um perfil de normalidade, mostrando certa assimetria, logo, optou-se por uma transformação logaritmica destes dados, o que atenuou razoavelmente o problema. Vale notar (Figuras 3 e 4), entretanto, que as correlações mostradas nestes diagramas de dispersão são um tanto prejudicadas pela presença de um ponto aberrante ou "outlier" correspondente a observação número 22 da TABELA 1. De modo a se ter uma melhor consistência nos dados, este aparente conflito entre esta observação excepcional e toda a massa de dados é atenuado substituindo-se este "outlier" pela média de seus dois pontos vizinhos. Com isto, obtevese uma descrição mais robusta e de melhor interpretabilidade destes dados, como pode ser observado pelos novos diagramas de dispersão (Figuras 3a e 4a), onde os novos coeficientes de explicação da DRC em função da porosidade são agora mais expressivos ainda (coeficiente de correlação passando de 0.63 na Figura 3 para 0.75 na Figura $3 a$ e de 0.61 na Figura 4 para 0.70 na Figura 4a).

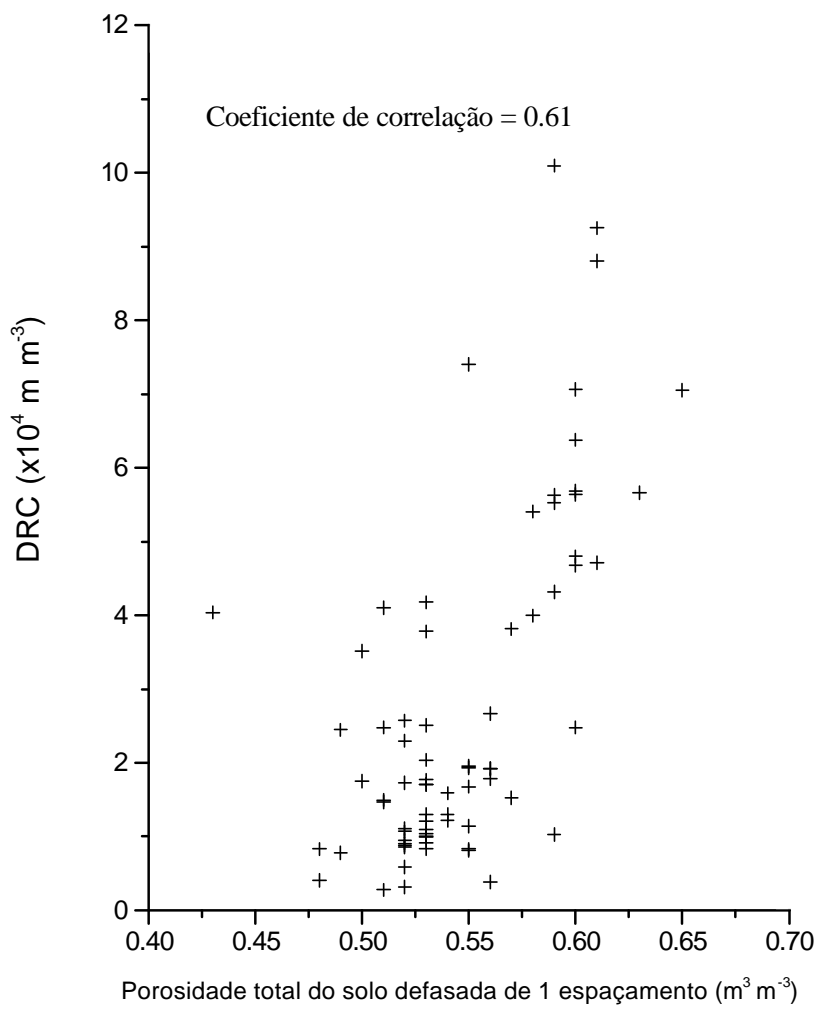

Figura 4 - A dispersão dos dados de comprimento de raiz em função da porosidade total do solo defasada de 1espaçamento, apresentando um coeficiente de correlação $(r)$ igual a 0.61 .

\section{Análise via modelos de espaço de estados (regressão dinâmica) versus regressão estática}

Para uma melhor visualização das características e vantagens da abordagem de espaço de estados (regressão dinâmica) no estudo da relação solo-planta (isto é, da relação $D R C \times$ porosidade do solo), é aqui apresentada, inicialmente e para efeito de comparação, uma análise clássica via modelos de regressão convencional (estática). Tais modelos de regressão tem como variável resposta a ser explicada o logaritmo da DRC (densidade radicular por comprimento de raiz) e como variáveis explicativas (regressores) a porosidade do solo e a porosidade do ponto vizinho. Neste modelo, os coeficientes de regressão $\beta_{1}$ e $\beta_{2}$ correspondentes a estas duas variáveis são os principais parâmetros de interesse, e medem o efeito ou variação esperada sobre a variável resposta em decorrência de uma variação unitária do regressor. Tais parâmetros, neste modelo estático são considerados fixos (constantes ao longo do espaço) e são aqui estimados, com base nos dados apresentados nas TABELAS 1 a 3, de dois modos diferentes: uma versão convencional (mínimos quadrados ordinários) e uma versão sequencial (mínimos quadrados sequenciais). Os resultados destas estimativas são apresentados na sessão seguinte (Figura 5). 


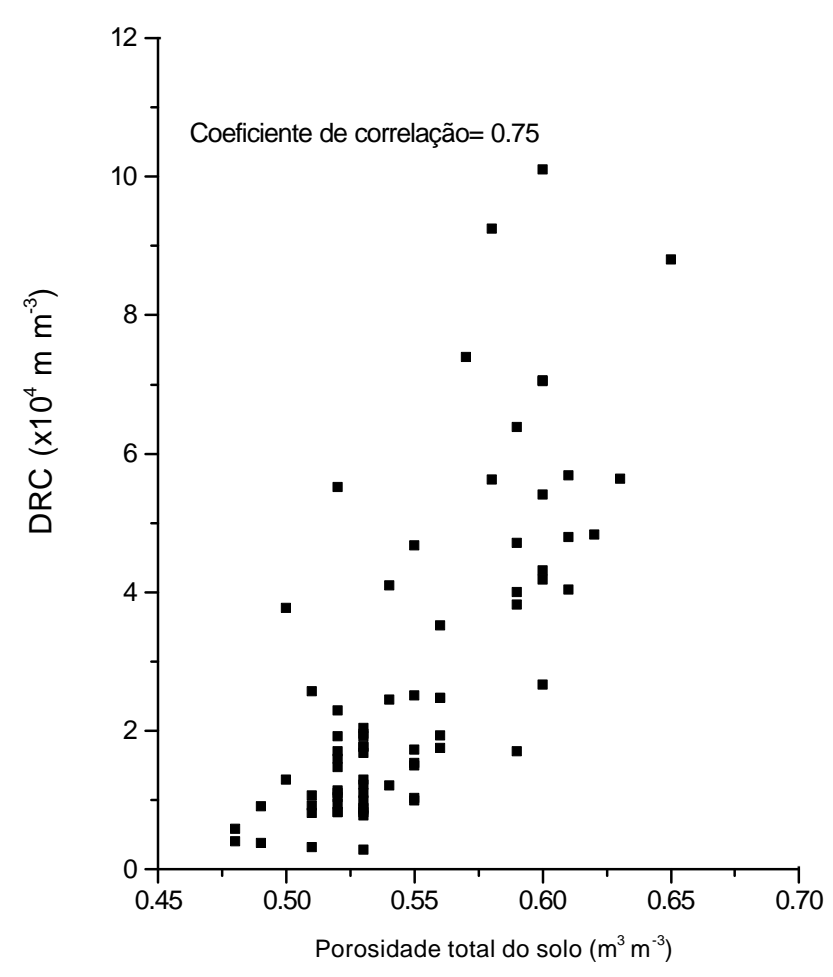

Figura 3a - A dispersão dos dados de comprimento de raiz em função da porosidade total do solo, apresentando um coeficiente de correlação (r) igual a 0.75 .

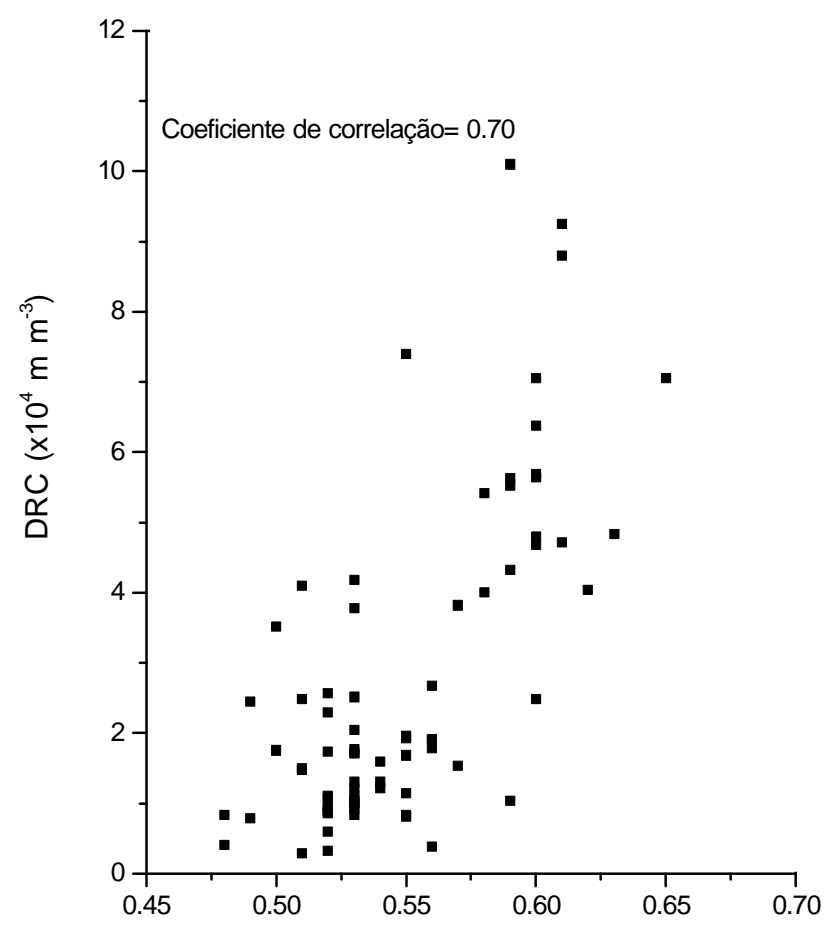

Porosidade total do solo defasada de 1 espaçamento $\left(\mathrm{m}^{3} \mathrm{~m}^{-3}\right)$

Figura 4a - A dispersão dos dados de comprimento de raiz em função da porosidade total do solo defasada de 1 espaçamento, apresentando um coeficiente de correlação $(r)$ igual a 0.70 .
Já no modelo de regressão dinâmica os coeficientes betas não são considerados fixos, mas variam ao longo do espaço segundo uma evolução markoviana (West \& Harrison, 1989, 1997; Barbosa, 1991) e são estimados por algoritmos tipo filtro de Kalman (versão ordinária e versão suavizada).

Utilizando o software BATS (Bayesian Analysis of Time Series) (West \& Harrison, 1989; Pole et al., 1994), procedeu-se a análise dos dados via modelos de regressão dinâmica representados em espaço de estados. As variáveis explanatórias consideradas neste modelo são as mesmas utilizadas no modelo estático. Desta forma, é aqui considerado o seguinte modelo de regressão dinâmica para a relação solo-planta, descrito pelas duas equações abaixo:

$(\text { LDRC })_{i}=\left(\beta_{0}\right)_{i}+\left(\beta_{1} \text { Poro }\right)_{i}+\left(\beta_{2} \text { Poro }\right)_{i-1}+(\text { Erro })_{i}$ equação das observações

$\left(\begin{array}{l}\beta_{0} \\ \beta_{1} \\ \beta_{2}\end{array}\right)_{i}=\left(\begin{array}{l}\beta_{0} \\ \beta_{1} \\ \beta_{2}\end{array}\right)_{i-1}+\left(\begin{array}{l}w_{0} \\ w_{1} \\ w_{2}\end{array}\right)_{i} \quad$ equação da evolução

onde:

$(\text { LDRC })_{i}=$ logaritmo da densidade radicular por comprimento de raiz no ponto i;

$\left(\beta_{0}\right)_{i}=$ componente de nível do processo no ponto i;

$\left(\beta_{1}\right)_{i}=$ coeficiente de regressão dinâmica (local) da variável porosidade no ponto i;

(Poro) $=$ porosidade total do solo no ponto $\mathrm{i}$;

$\left(\beta_{2}\right)_{i}=$ coeficiente de regressão dinâmica (local) da variável porosidade no ponto $\mathrm{i}-1$, ou seja, defasada de 1 espaçamento;

(Poro $_{\mathrm{i}-1}=$ porosidade total do solo no ponto $\mathrm{i}-1$;

$($ Erro $)=$ componente de erro observacional (aleatório) no ponto i, com média zero, variância $v_{i}$ e distribuição normal; vetor $w_{i}=\left(\begin{array}{lll}w_{0} & w_{1} & w_{2}\end{array}\right)^{\prime}$; das perturbações do sistema, com média zero, variância constante e distribuição normal.

\section{RESULTADOS E DISCUSSÃO}

Na Figura 5 são apresentadas as estimativas dos coeficientes do modelo de regressão estática, tanto em estimativas constantes como em estimativas sequenciais. O último valor das estimativas sequenciais de cada coeficiente coincide com as respectivas estimativas fixas (não-sequenciais). Estas estimativas sequenciais, além de englobarem as estimativas fixas como um de seus pontos (o último) são obtidas pelo algoritmo de mínimos quadrados sequenciais, que é um caso particular do algoritmo do filtro de Kalman com implementação via fatores de desconto, tomando estes fatores iguais a 1.00. Uma diferença importante entre estas duas versões do modelo estático reside em apresentarem valores ajustados diferentes, e, em consequência diferentes coeficientes $R^{2}$ de ajuste, em favor da versão sequencial, como pode ser visto nas Figuras $5 a$ e $5 b\left(R^{2}=0.69\right.$ para versão 
sequencial e $R^{2}=0.59$ para versão tradicional). Este modelo estático e sua correspondente capacidade de explicação dos dados é aqui tomado como referência para comparação com os modelos dinâmicos.

São apresentadas as estimativas dos coeficientes de um modelo de regressão dinâmica (Figuras 6a e 6b) onde é permitida uma variabilidade de $1 \%$ para os coeficientes de regressão (entre cada dois pontos consecutivos), o que corresponde a um fator de desconto

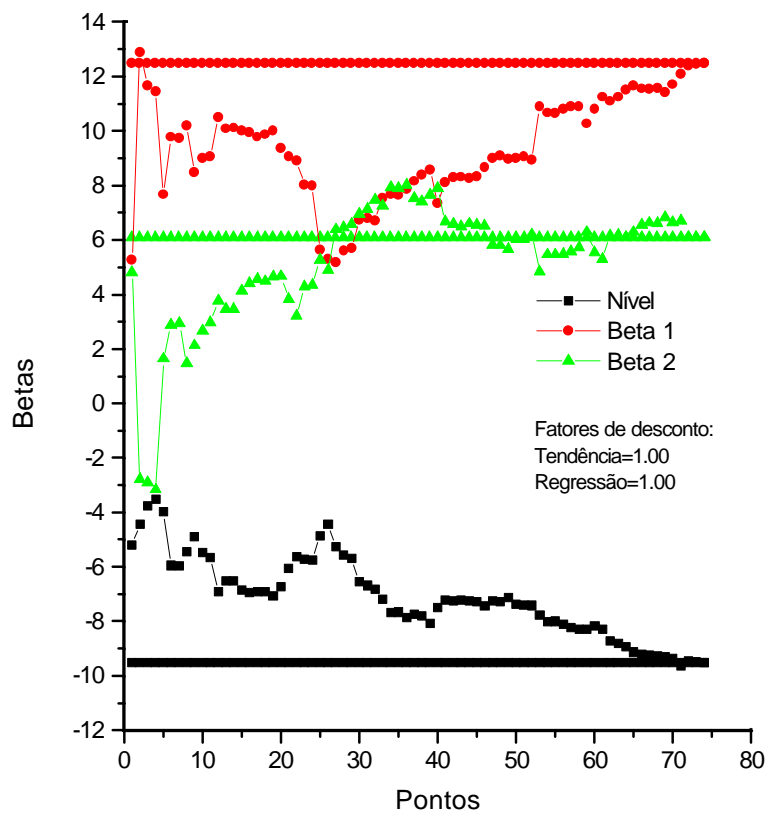

Figura 5 - Modelo de regressão estática: estimativas sequenciais dos coeficientes versus estimativas constantes.

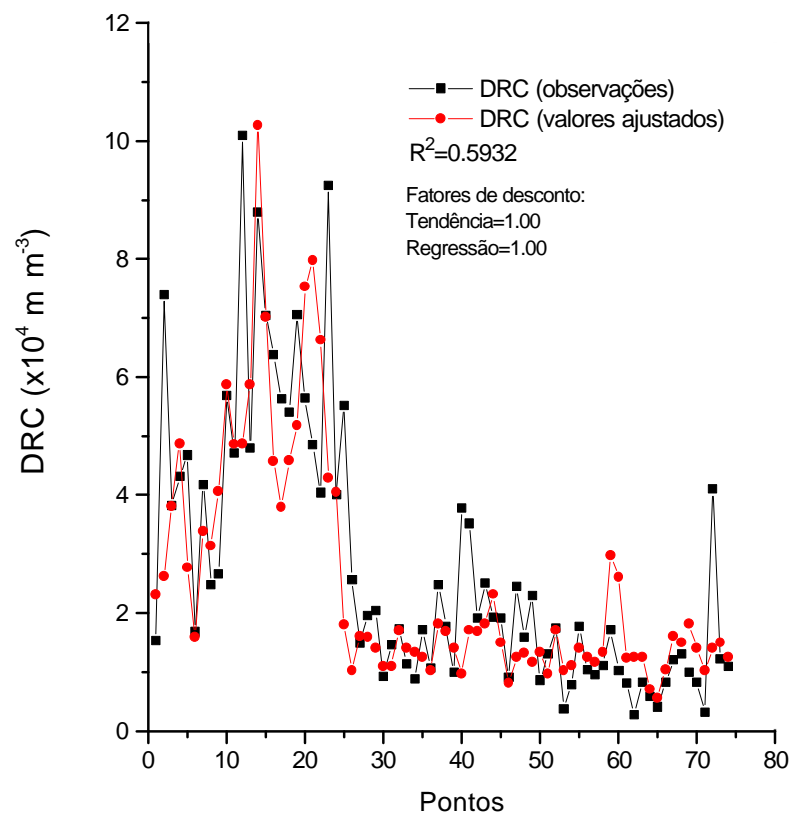

Figura 5a - Observações de DRC versus valores ajustados pelo modelo de regressão estática usando estimativas constantes dos coeficientes. no filtro de Kalman (FK) de 0.99. Com isto, o modelo de regressão tornou-se ligeiramente dinâmico (tanto na versão ordinária do FK dada na Figura 6a quanto na versão suavizada do FK dada na Figura 6b). Já para o coeficiente de nível do processo, foi considerada uma maior dinamicidade (fator de desconto de 0.95), resultando no conjunto em um coeficiente de explicação em torno de 0.98 a 0.99 (Figuras $6 c$ e $6 d$ ), o que representa uma melhora expressiva em relação ao modelo estático, mesmo quando considerado em sua versão sequencial. Vale ressaltar as vantagens da versão suavizada em relação a versão ordinária do modelo dinâmico, pois embora com uma pequena redução no grau de explicação dos dados (de 0.99 para 0.98 ), tem-se uma melhor interpretabilidade para os coeficientes estimados na versão suavizada, por apresentarem menor variabilidade, como constatado nas Figuras 6a e 6b.

Vale mencionar que a escolha precisa do grau de dinamicidade do modelo de regressão dinâmica pode ser automatizada através do uso de softwares adequados para a implementação destes modelos. Assim, por exemplo, no caso do software BATS aqui utilizado são tomados como valores de referência ("default") para os fatores de desconto dos coeficientes de regressão o valor de 0.98 e 0.90 para o nível do processo (chamado de tendência), produzindo os resultados apresentados nas Figuras de $7 \mathrm{a}$ a $7 \mathrm{~d}$, o que difere muito pouco dos resultados do modelo dinâmico anterior. Com isto, notase que não há dificuldade de ordem prática na implementação destes modelos, com expressivas vantagens em relação aos modelos estáticos de regressão convencionais.

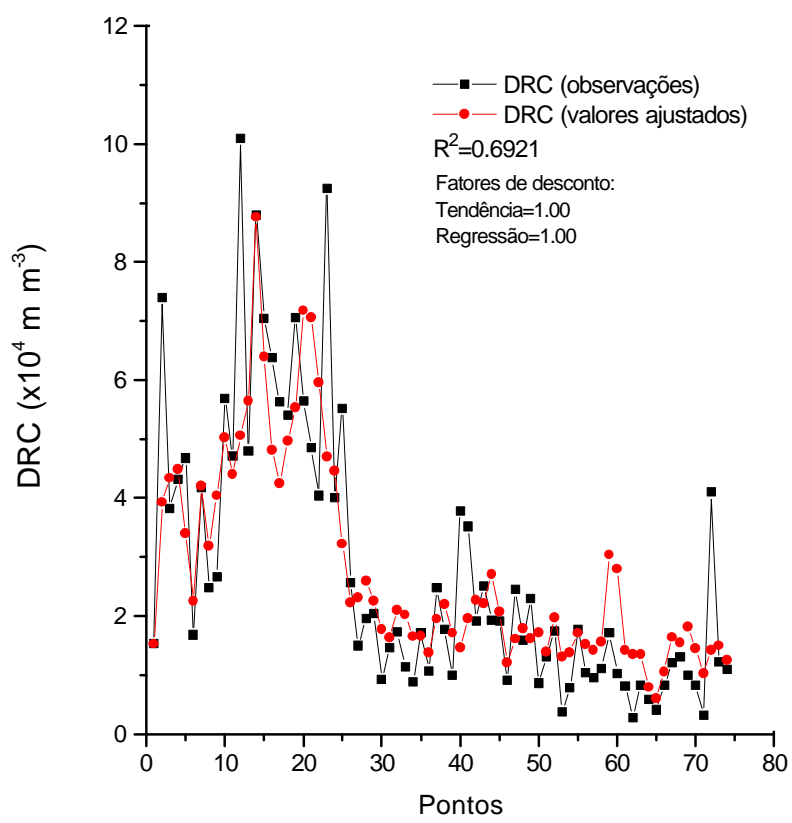

Figura 5b - Observações de DRC versus valores ajustados pelo modelo de regressão estática usando estimativas sequenciais dos coeficientes. 


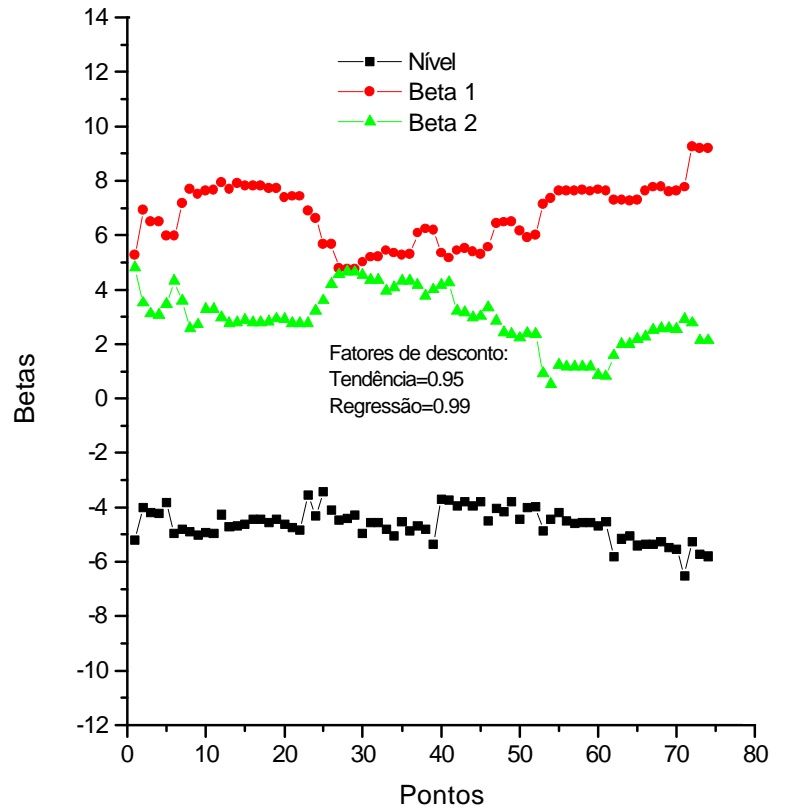

Figura 6a - Modelo de regressão dinâmica (espaço de estado): estimativas sequenciais dos coeficientes.

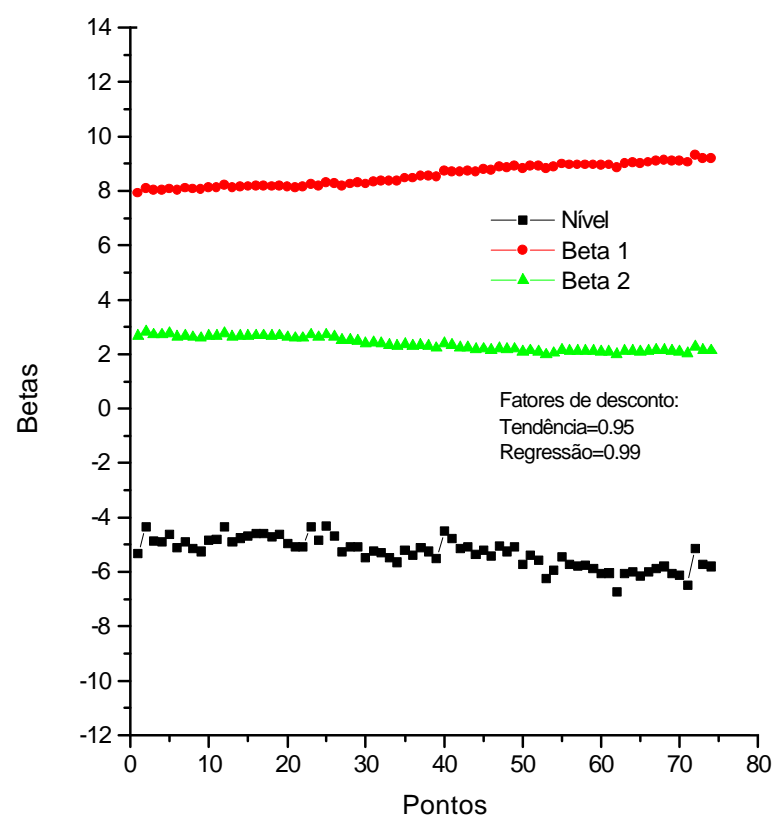

Figura 6b - Modelo de regressão dinâmica (espaço de estado): estimativas sequenciais suavizadas dos coeficientes.

O uso de modelos de regressão dinâmica, representados em espaço de estados, para quantificar as relações solo-planta têm sido pouco explorado na literatura, principalmente por se tratar de um assunto relativamente recente (embora introduzidos originalmente na década de 60, só a partir dos anos 80 sua implementação prática se tornou disseminada), em que foi introduzida uma abordagem prática de implementação (West \& Harrison, 1989, 1997), facilitando o uso destes modelos. Pelo fato de serem modelos de ajuste local,

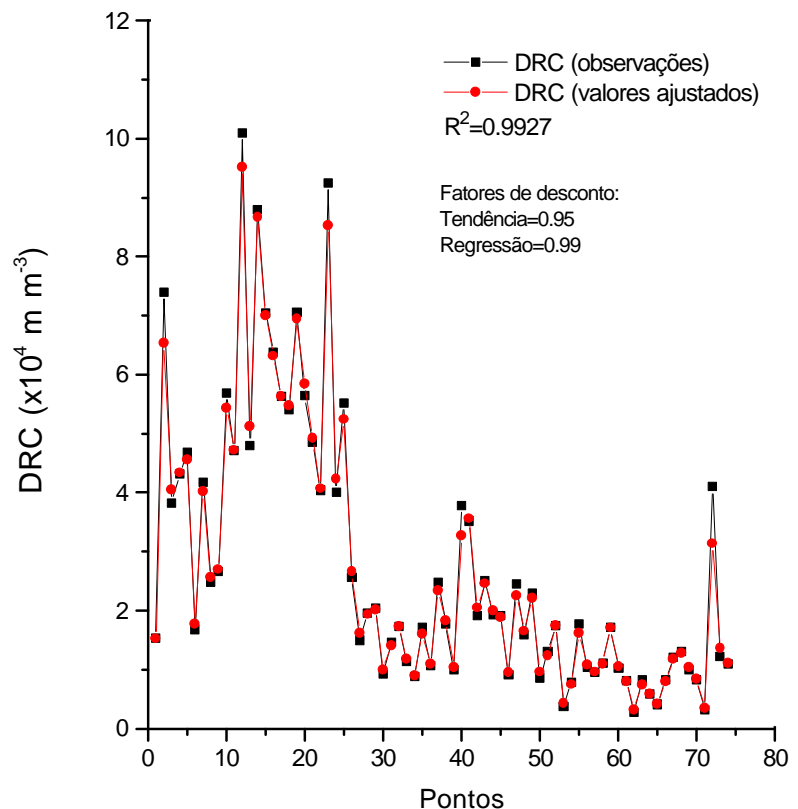

Figura 6c - Observações de DRC versus valores ajustados pelo modelo de regressão dinâmica (espaço de estado): versão ordinária.

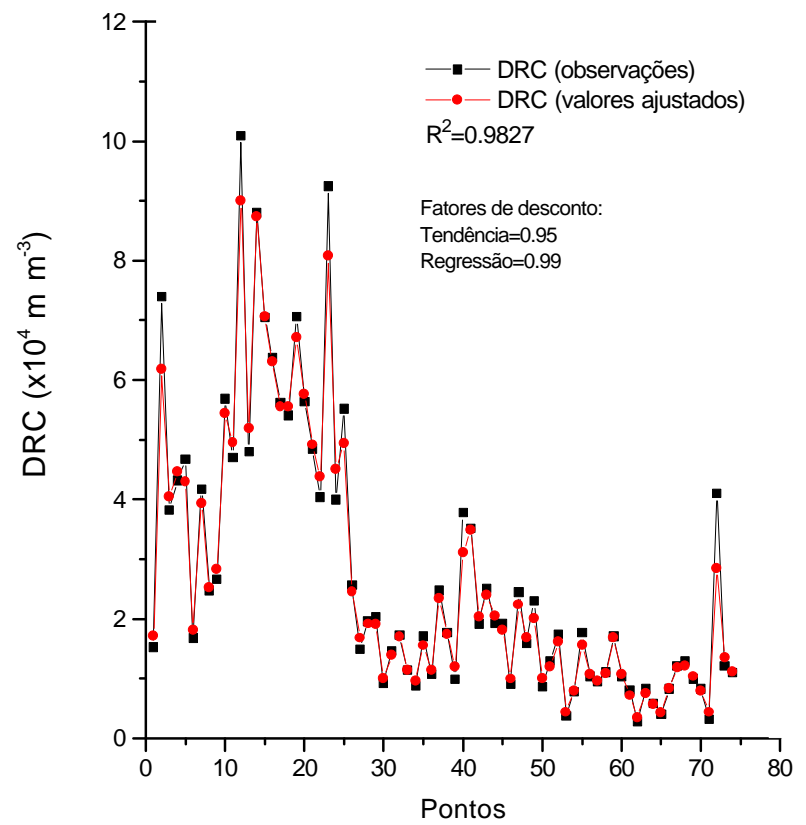

Figura 6d - Observações de DRC versus valores ajustados pelo modelo de regressão dinâmica (espaço de estado): versão suavizada.

torna-se possível estimar os coeficientes de regressão da(s) variável(is) em cada ponto de amostragem, obtendose desta forma, uma estimativa mais precisa do comportamento da variável de interesse, o que ameniza o problema da variabilidade espacial, principalmente quando se tem o interesse de aplicar uma agricultura de precisão. Por outro lado, os modelos de regressão estáticos, que normalmente são utilizados, são modelos de ajuste global, ou seja, seus coeficientes de regressão são valores médios que não variam ao longo do espaço. 


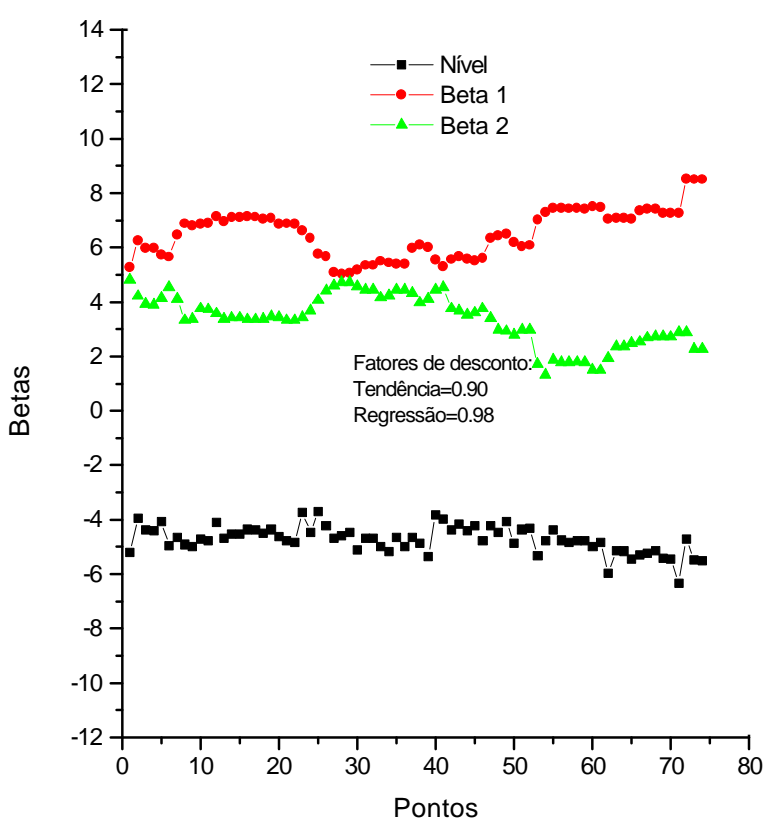

Figura 7a - Modelo de regressão dinâmica (espaço de estado): estimativas sequenciais dos coeficientes.

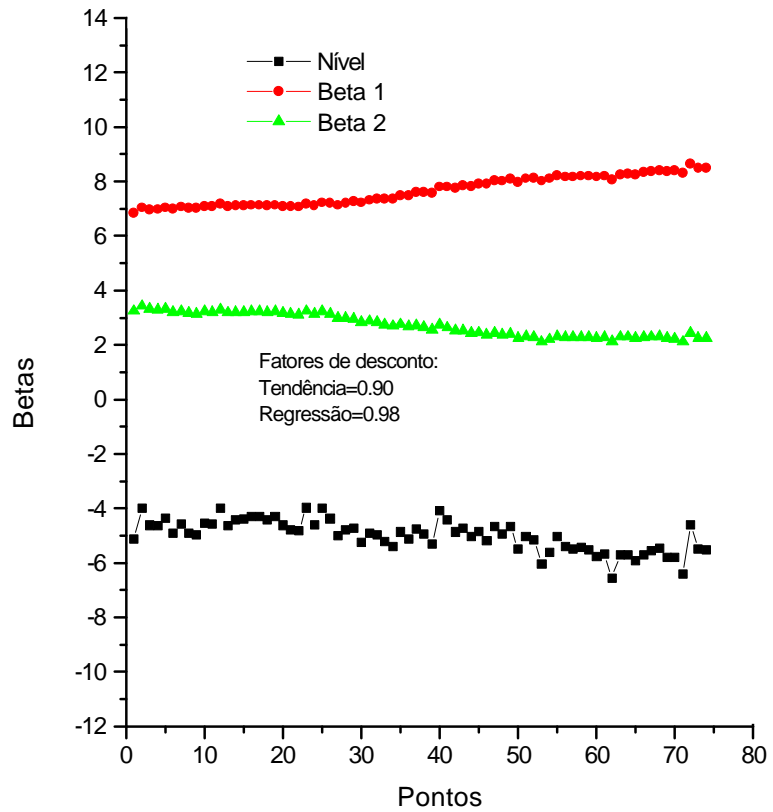

Figura 7b - Modelo de regressão dinâmica (espaço de estado): estimativas sequenciais suavizadas dos coeficientes.

Em função disto, verificou-se por intermédio deste estudo que a aplicação dos modelos de regressão dinâmica (espaço de estado) se adequam melhor ao estudo das relações solo-planta em virtude do carácter local desse relacionamento.

\section{AGRADECIMENTOS}

À FAPESP e ao CNPq pelo suporte financeiro a este projeto.

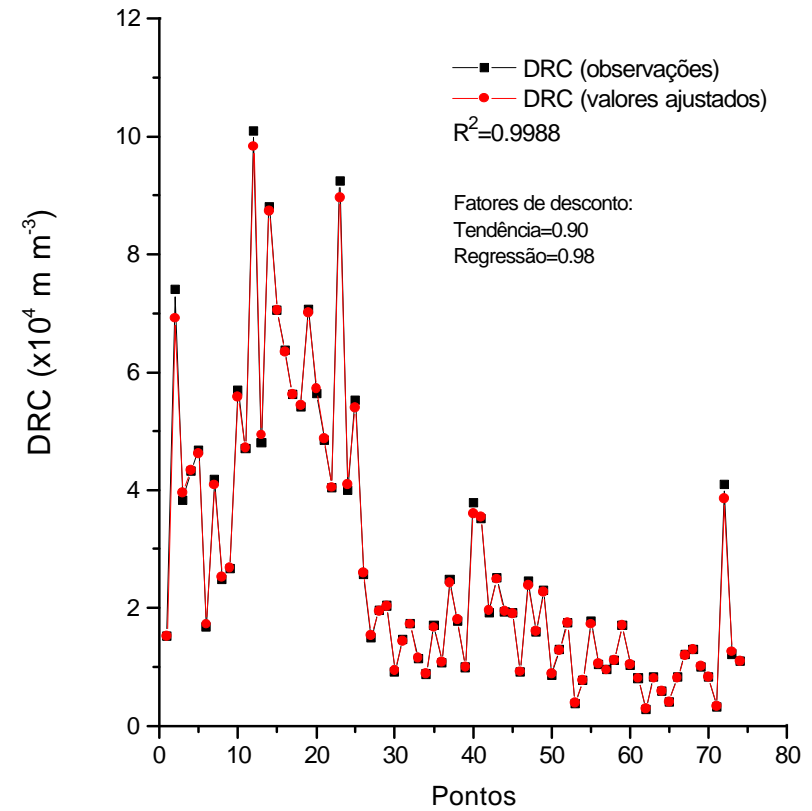

Figura 7c - Observações de DRC versus valores ajustados pelo modelo de regressão dinâmica (espaço de estado): versão ordinária.

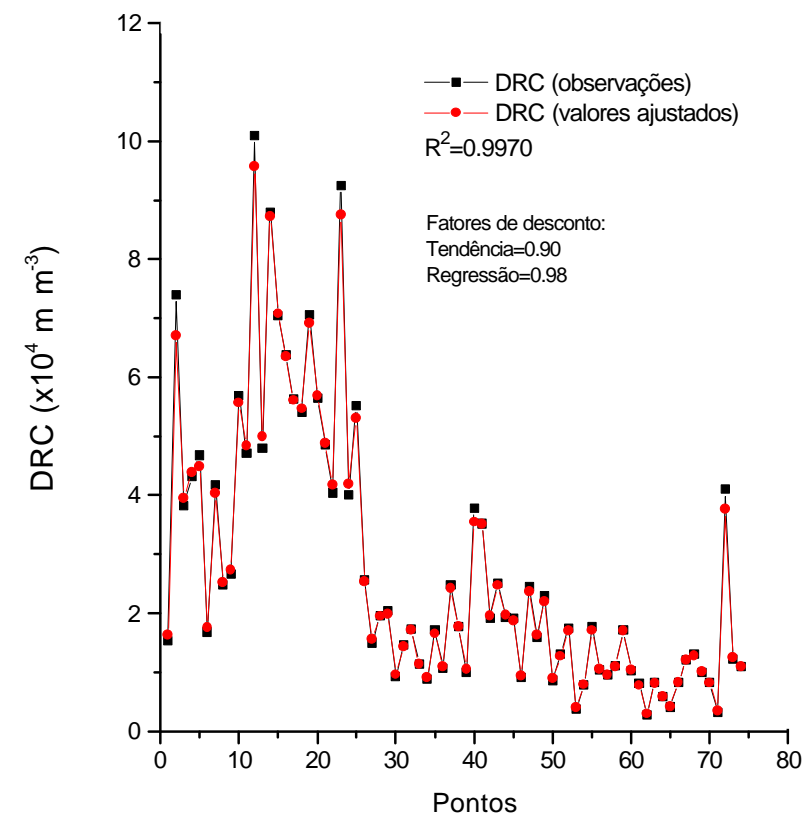

Figura 7d - Observações de DRC versus valores ajustados pelo modelo de regressão dinâmica (espaço de estado): versão suavizada.

\section{REFERÊNCIAS BIBLIOGRÁFICAS}

BARBOSA, E.P. Séries temporais múltiplas: uma análise via modelos lineares dinâmicos., Rio de Janeiro: IMPA,1991. /Mini-curso apresentado na $4^{\text {a }}$ Escola de Séries Temporais/ BOHM, W. Methods of studying root systems. New York: Springer-Verlag, 1979. 189p.

BROWN. D.A.; SCOTT, H. don. Dependence of crop growth and yield on root development and activity. In: BARBER, S.A.; BOULDIN, D.R. (Ed.) Roots and water influx, and plant growth. Madison: Soil Science Society of America, 1984. cap.6, p.101-136. 
CRESTANA, S.; GUIMARÃES, M.F.; JORGE, L.A.C.; RALISH, R.; TOZZI, C.L.; TORRE, A.; VAZ, C.M.P. Avaliação da distribuição de raízes no solo auxiliada por processamento de imagens digitais. Revista Brasileira de Ciência do Solo, v.18, p.365-371, 1994.

DOURADO-NETO, D.; TIMM, L.C.; OLIVEIRA, J.C.M.; REICHARDT, K.; BACCHI, O.O.S.; TOMINAGA, T.T.; CASSARO, F.A.M. State-space approach for the analysis of soil water content and temperature in a sugarcane crop. Scientia Agricola, v.56, p.1215-1221, 1999. Suplemento.

EMPRESA BRASILEIRA DE PESQUISA AGROPECUÁRIA. Centro Nacional de Pesquisa de Solo. Manual de métodos de análise de solos. 2.ed. Rio de Janeiro, EMBRAPA, CNPS, 1997. 212p.

FANTE JÚNIOR, L. Sistema radicular da aveia forrageira avaliado por diferentes métodos, incluindo processamento de imagens digitais. Piracicaba, 1997. 119p. Tese (Doutorado) - Centro de Energia Nuclear na Agricultura, Universidade de São Paulo.

FANTE JÚNIOR, L.; REICHARDT, K.; JORGE, L.A.C.; BACCHI, O.O.S. Distribuição do sistema radicular de uma cultura de aveia forrageira. Scientia Agricola, v.56, p.1091-1100, 1999. Suplemento.

HATANO, R.; IWANAGA, K.; OKAJIMA, H.; SAKUMA, T. Relationship between the distribution of soil macropores and root elongation. Soil Science and Plant Nutrition, v.34, p.535-546, 1988.

HUI, S.; WENDROTH, O.; PARLANGE, M. B.; NIELSEN, D. R. Soil variability: infiltration relationships of agroecosystems. Journal of Balkan Ecology, v.1, p.21-40, 1998.

KIEHL, E.J. Manual de edafologia: relações solo-planta. São Paulo: Agronômica Ceres, 1979. 246p.

KOPKE, U. Methods for studying root growth. In: SYMPOSIUM ON THE SOIL/ROOT SYSTEM IN RELATION TO BRAZILIAN AGRICULTURE, Londrina, 1980. Proceedings. Londrina: Fundação Instituto Agronômico do Paraná, 1981. p.303-318.
LONGSDON, S.D.; LINDEN, D.R. Interactions of earthworms withsoil physical conditions influencing plant growth. Soil Science, v.154, p.330-337, 1992.

MEROTTO JÚNIOR, A.; MUNDSTOCK, C.M. Wheat root growth as affected by soil strength. Revista Brasileira de Ciência do Solo, v.23, p.197-202, 1999.

MORKOC, F.; BIGGAR, J. W.; NIELSEN, D. R.; ROLSTON, D. E. Analysis of soil water content and temperature using Statespace approach. Soil Science Society of America Journal, v.49, p.798-803, 1985.

PARLANGE, J.Y.; LISLE, I.G.; BRADDOCK, R.D.; SMITH, R.E. The three-parameter infiltration equation. Soil Science, v.133, p.337-341, 1992.

POLE, A.; WEST, M.; HARRISON, J. Applied bayesian forecasting and time series analysis. London: Chapman \& Hall, 1994. 409p.

SALIH, A.A.; BABIKIR, H.M.; ALI, S.A.M. Preliminary abservations on affects of tillage systems on soil physical properties, cotton root growth and yield in Gezira Scheme, Sudan. Soil Tillage Research, v.46, p.187-191, 1998.

SHUMWAY, R.H. Applied statistical time series analysis. Englewood Clifs: Prentice-Hall, 1988. 379p.

TERUEL, D.A. Caracterização arquitetural do sistema radicular de soja. Piracicaba, 1999. 106p. Tese (Doutorado) - Escola Superior de Agricultura "Luiz de Queiroz", Universidade de São Paulo.

WENDROTH, O.; AL OMRAN, A. M.; KIRDA, K.; REICHARDT, K.; NIELSEN, D. R. State-space approach to spatial varialility of crop yiel. Soil Science Society of America Journal, v.56, p.801-807, 1992.

WEST, M.; HARRISON, J. Bayesian forecasting and dynamic models. London: Springer-Verlag, 1989. 704p.

WEST, M.; HARRISON, J. Bayesian Forecasting and Dynamic Models. Springer-Verlag, 1997. 681p.

Recebido em 10.01 .00 